\title{
La Oscilación del Atlántico Norte: un fenómeno que incide en la variabilidad climática de México
}

\author{
N. Sánchez-Santillán*, M. Signoret-Poillon* y R. Garduño-López ** \\ Departamento el Hombre y su Ambiente, UAM-Xochimilco*, Departamento de Climatología del \\ Centro de Ciencias de la A tmósfera, UNAM** \\ E-mail: santilla@ correo.xoc.uam.mx
}

(recibido: abril de 2005; aceptado: agosto de 2005)

\begin{abstract}
Resumen
La Oscilación del Atlántico Norte es un fenómeno climático que afecta a esta región de la Tierra, pero por efecto de las teleconexiones, influye en zonas que se encuentran mucho más allá de su área de acción directa, como son las aguas tropicales de la India y el Océano Pacífico. Las actuales investigaciones sobre este evento, tratan de establecer su prob able relación con el ENOS (EI Niño-Oscilación del Sur), además del impacto que en su periodicidad e intensidad tiene la creciente concentración atmosférica de los gases de invernadero. Se analizan también los factores de la dinámica de la atmósfera que conciernen al fenómeno, como son la energía solar, el movimiento de grandes masas de aire y la presión barométrica.
\end{abstract}

Descriptores: Oscilación del Atlántico Norte, variabilidad climática.

\begin{abstract}
TheN orth At lan tic 0 scil la tion is a cli ma ticphenomenon that affectsthis region of the Earth, but that by ef fect of teleconnections, in flu ences ar eas lo cated far beyond its area of di rect action, Such as the trop i cal wa ters of In dia and the Pa cific $O$ cean. Cur rent re search on this event try to es tablish its likely relationship with the EN SO ( $\mathrm{E} / \mathrm{N}$ iño/S outh ern $\mathrm{O}$ s cil la tion), be sides the im pact that thegrow ing atmo spheric con cen tra tion of green househas on its rhythm and intensty. At mo spheredy nam ics factors con cern ing thephenomenon, such as solar en ergy, movement of largeair massesand at mo spheric pres. sureareal so an a lyzed.
\end{abstract}

Key words: N orth Atlan ticO scil lation, cl maticvariabil ity.

\section{Introducción}

La Oscilación del Atlántico Norte (OAN) es un fenómeno atmosférico de ocurrencia periódica, cuya naturaleza es semejante a la del ENOS (EI NiñoOscilación del Sur), aunque mucho menos conocido; de hecho, la primera descripción científica oficial de ambos eventos se debe a la misma persona, el meteorólogo inglés Sir Gilbert Thomas Wal ker, quien lo publicó en un artículo en 1923.

A pesar del reducido número de estudios de los que la OAN ha sido objeto desde entonces, ha cobrado importancia a últimas fechas entre la comunidad científica especializada, por nuevas razones que se añaden a la del crucial efecto que su dinámica ejerce sobre la variabilidad climática de las regiones que abarca, es decir, aquéllas que limitan con la porción Norte del océano Atlántico, como Norte América, (México, Estados Unidos y Canadá) y en el otro extremo, con las islas y la parte de continente que conforma Europa Noroccidental.

Uno de los factores que ha puesto en boga el tema de la OAN es la probable relación de una buena parte de su comportamiento actual con la 
La Oscilación del Atlántico Norte: un fenómeno que incide en la variabilidad ...

concentración de $\mathrm{CO}_{2}$ en la atmósfera; otro más, es la posibilidad de que su influencia sobre el clima mundial comprenda otras zonas más allá del Atlántico Norte, debido a su inminente vinculación con el evento de El Niño y, por otro lado, al efecto de las teleconexiones. De cualquier manera, las investigaciones en este sentido aún son incipientes, y con el tiempo, se determinará la verdadera dimensión del fenómeno.

Es importante mencionar, que al estar la República Mexicana dentro de la zona de influencia de la OAN, los resultados de los estudios arrojaron información crítica para explicar las variaciones climáticas en nuestro país y poder pronosticar a corto y largo plazos, entre otros importantes sucesos, el comportamiento de las temporadas de lluvias, de secas y el rigor de las temperaturas de invierno y verano.

Por ser la OAN, básicamente, un fenómeno de movimiento de grandes masas de aire atmosférico en una escala in ter con ti nen tal, con un impacto decisivo en la climatología, se ha considerado pertinente en esta contribución, introducir algunas breves pautas de la dinámica de la atmósfera y el clima para la mejor comprensión del tema que aquí se trata.

\section{Antecedentes: Climatología y teleconexiones}

Para entender la climatología de una región no basta con conocer sólo los patrones de comportamiento de los elementos climáticos, (temperatura, lluvia y humedad, dirección y fuerza del viento, presión atmosférica, nubosidad, radiación solar y visibilidad), o los factores climáticos, (latitud, altitud, relieve, distribución de tierras y aguas, y corrientesmarinas).

Hasta hace unas cuantas décadas, el clima era considerado por gran parte de los biólogos, como un fac tor abiótico con carácter estático y con una dinámica, aparentemente, bien establecida. Si bien, el clima evolucionaba, las escalas de tiempo en las que se creía también se manifestaban; estos procesos ocurrían en una escala de miles de años. Sin embargo, pocas veces se hizo el esfuerzo de explicar su comportamiento a partir de las relaciones dinámicas existentes entre el océano y la atmósfera como un sistema acoplado, así como la relación que este binomio guardaba con la superficie terrestre, la criosfera y a la biosfera.

En la actualidad, la climatología se aborda como un sistema dinámico altamente complejo, que opera en diferentes escalas de tiempo y espacio. Esto, aunado a los poderosos sistemas de cómputo que facilitan el manejo de una gran cantidad de datos, está facilitando la transición en la forma de comprender y abordar los diversos fenómenos que involucran, tanto la climatología de una región, como la variabilidad inherente a ella, y que inicia, en la mayoría de los casos, con una dependencia crítica de las condiciones iniciales de los procesos analizados, la cual puede desencadenar eventos de teleconexión, es decir, de enlaces a grandes distancias entre anomalías climáticas aparentemente desconectadas entre sí (Glantzet al., 1991).

Uno de los eventos de teleconexión climática más conocido actualmente es el ENOS (EI Niño/ Oscilación del Sur), al que se ha responsabilizado de la variabilidad, tanto de la lluvia y la temperatura en múltiples regiones del planeta, como de algunas respuestas de la biosfera que se han observado a partir del estudio de poblaciones puntuales. Este evento -antes considerado como fenómeno, por el desconocimiento que se tenía de él- constituye una de las mejores herramientas para explicar la variabilidad y la dinámica del clima, tanto a escala local como global, en períodos de entre 3 y 5 años (Cook, 1992; Díaz y Kiladis, 1992; Nicholls, 1992; Sánchez, 1999).

La Oscilación del Atlántico Norte (OAN), es un evento climático que ha ocurrido desde hace miles de años, pero no contaba con una explicación amplia y detallada de su magnitud, origen y periodicidad, ni de su posible impacto en estudios de caso. Tan sólo entre 1981 y 2000 se contaba con apenas un centenar de publicaciones realizadas en todo el mundo que abordaban este fenómeno, cantidad que contrasta con las miles que en ese mismo período se han escrito con respecto al evento de El Niño (Stephensonet al., 2002).

\section{Energía solar, motor de los movimientos de las masas de aire}

La atmósfera húmeda no solamente atrapa en la Tierra la radiación infrarroja que emite luego de ser 
N. Sánchez-Santillán, M. Signoret-Poillon y R. Garduño-López

calentada por la energía del Sol, sino que también almacena energía. El vapor de agua movido por los vientos, es uno de los vehículos más importantes de la atmósfera para el transporte del calor. Cuando la energía solar alcanza este vapor de agua, la masa de aire se calienta desde abajo, ya que la exposición a nivel de la superficie de esta energía es particularmente intensa. Los millones de toneladas de agua que se elevan desde los océanos del mundo, representan innumerables moléculas en un movimiento tan violento, que se han separado de sus compañeras, dando paso a que el agua líquida se convierta en gas (Flohn, 1968).

Este vapor existe en cierta proporción en todos los cielos. La inmensa cantidad de energía que lleva consigo se denomina "calor latente", que se convierte en calor sensible cuando el vapor de agua vuelve a condensarse transformándose en líquido. Cada vez que el aire se enfría lo suficiente, su vapor se condensa en forma de nubes y el calor liberado pasa a la atmósfera (Schaefer y Day, 1981).

El aire frío de latitudes templadas y polares contiene poca de esta energía latente, pero los vientos de los trópicos, cargados de humedad, transportan grandes cantidades de calor latente al desplazarse hacia el polo, donde al enfriarse se precipitan en forma de copiosas lluvias 0 , incluso, de nevadas. Algunos meteorólogos han estimado que $25 \mathrm{~mm}$ de lluvia precipitados sobre una superficie, liberan la energía equivalente a tres días de Sol sobre esa misma área (Durand, 1969).

El aire húmedo transporta grandes cantidades de calor latente; el trabajo de la máquina atmosférica consiste en distribuir ese suministro térmico en movimiento. La atmósfera efectúa la tarea de intercambiar el calor por movimientos verticales del aire, mediante un proceso denominado convección y, en un sentido horizon tal, a través de la advección.

\section{El papel de la presión barométrica en el movimiento de las masas de aire}

Cuando el aire aumenta su temperatura asciende verticalmente; éste se mueve porque algunas de sus par tes tienen más energía que otras, es decir, están más calientes que otras. En ese momento, sus moléculas se agitan y se empujan, apartándose mutuamente. El aire se dilata y, al expandirse, el número de sus moléculas disminuye por centímetro cúbico. La presión atmosférica en un punto determinado es la medida del peso total de aire por encima de él. Debido a su menor densidad, una columna de aire caliente pesa menos que una columna igual pero de aire frío, y ejerce entonces, menos presión.

Las diferencias de temperatura determinan desequilibrios de presión y a menos que intervenga un fac tor extraño, por ejemplo, una cadena montañosa, los desequilibrios horizontales barométricos ocasionan, inexorablemente, un desplazamiento de aire del área de mayor presión a la de menor presión.

\section{Un ciclo sin fin}

En la atmósfera, los ciclos de desplazamiento de las masas de aire se producen a gran escala entre las regiones ecuatoriales y las superficies gélidas de los polos, de un modo que dista mucho de ser sencillo. El aire es más caliente alrededor de la franja ecuatorial del planeta, por ser el lugar al que va a parar la mayor parte de la energía solar y en el cual el vapor de agua captura más calor. Es allí donde toda la descripción del tiempo atmosférico y del clima comienza su vertiginoso viaje.

El aire al dilatarse es empujado hacia arriba hasta alcanzar la tropopausa, límite entre la troposfera y la estratosfera, y se extiende en forma horizontal hacia los polos (el Norte o el Sur) del planeta. Muy por debajo, el aire frío se mueve hacia el Ecuador para ocupar el lugar que aquél deso cupó. La masa de aire caliente, de entre 8 y 16 $\mathrm{km}$ de altura, se va desplazando hacia fuera, en dirección a las regiones de menor presión. Enfriándose y hundiéndose, realiza un largo y aventurado viaje, descendiendo de nuevo a la superficie terrestre, en las latitudes polares donde por efectos térmicos predomina la alta presión. En ese momento, fría, densa, seca y lenta, la masa de aire comienza a moverse de regreso hacia el Ecuador. A esta máquina térmica se le denomina celda de Hadley; sin embargo, la rotación de la Tierra modifica este simplificado esquema general, fraccionando dicha celda en una serie de circulaciones 
La Oscilación del Atlántico Norte: un fenómeno que incide en la variabilidad ...

mucho más complejas. Asimismo, el globo terrestre dista de ser una superficie homo génea debido a la existencia de océanos y continentes heterogéneamente distribuidos, razón por la cual, las zonas de alta y baja presión no son franjas continuas, sino por el contrario, están conformadas por una serie de celdas de alta y baja presión. Sin em bargo, la situación se complica aún más con las estaciones del año, y esta consecuencia se refleja en el desplazamiento de la enorme máquina atmosférica hacia el Norte du rante el verano y hacia el Sur en el invierno (Durand, 1969).

La convección desplaza el calor de los trópicos a los casquetes polares, así como a innumerables lugares intermedios. Ejemplos de la convección son la brisa marina que sopla hacia el cálido in te rior du rante el día y la brisa terrestre que de noche se encamina hacia el mar, cuando la tierra ha irradiado gran parte de su calor. La convección en el día, hace subir el aire a una montaña abrasada por el sol, y por la noche, hace bajar el aire fresco de la montaña al caldeado valle. El efecto de la convección consiste en desplazar aire de los lugares fríos a los calientes y transferir calor de los calientes a los fríos.

No obstante, a pesar de lo sencillo que pueden parecer estos ejemplos de circulación por convección, los vientos, la orografía, la distribución de océanos y continentes, junto con la latitud y la altitud, alteran por completo el patrón. Las presiones del aire distan mucho de estar regularmente graduadas al Norte y Sur del Ecuador y las diferencias entre la distribución de tierras y aguas, además del día y la noche, determinan variaciones bruscas de temperatura. Por otro lado, debido a la rotación de la Tierra, los vientos que tienden a ir de los centros de alta presión a los de baja registran desviaciones; esto se explica de la siguiente forma: si una partícula de aire se mueve de un paralelo geográfico mayor a uno menor se desviará hacia el Este, debido a que la velocidad lineal en el paralelo mayor es más alta que en el menor, por lo que se adelanta, es decir, se desplaza en el sentido que gira la Tierra, que es de Oeste a Este. Por otra parte, si el viento va de un paralelo menor a uno mayor, debido a la velocidad lineal más baja en el paralelo menor, queda retardado con respecto a la velocidad del paralelo mayor desviándose en sentido contrario al que gira la Tierra, es decir, se desvía hacia el Oeste. Por otro lado, la rotación de la Tierra desvía las corrientes de aire de Norte a Oeste, respectivamente (García, 1978).

No existen entonces, capas de aire lisas y homogéneas en la atmósfera, que fluyan majestuosamente del Ecuador a los polos y de vuelta, más bien, el aire es descarrilado la mayoría de las veces por irregularidades accidentales. Se engendran masas de aire que chocan y se mezclan entre sí, y el intercambio de calor ocurre entre agitadas corrientes, rápidos remolinos y estallidos de la siempre inquieta atmósfera.

\section{Masas de aire: una unidad atmosférica}

Las masas de aire son las unidades atmosféricas de mayor magnitud que se conocen. Si una de ellas se cierne durante suficiente tiempo sobre alguna porción geográfica que puede ser un océano, una cordillera 0 una planicie, por mencionar sólo tres, tiende a adquirir las características de humedad y temperatura de la superficie que tiene debajo. Pueden cubrir varios millones de kilómetros cuadrados y es posible describirlas como volúmenes coherentes, porque su temperatura y contenido de humedad son bastante uniformes a una altura determinada y además, porque cuando empiezan a desplazarse lo hacen en conjunto (Schaefer y Day, 1981).

Las masas de aire se encuentran entre las partes móviles más importantes de la máquina atmosférica. Se originan en unas 20 regiones distribuidas en todo el mundo a las que se denominan centros de alta o baja presión; los primeros se sitúan principalmente sobre los océanos, y el viento en ellos gira de dentro hacia fuera, como en el sentido de las manecillas del reloj, en el Hemisferio Norte; en los segundos, ubicados sobre las porciones continentales, el viento gira de afuera hacia adentro, contrario a las manecillas del reloj, en el mismo Hemisferio.

Las masas que se dirigen al Norte se encuentran y actúan recíprocamente con las que se dirigen hacia el Sur, en las zonas templadas, dando 
N. Sánchez-Santillán, M. Signoret-Poillon y R. Garduño-López

a las diversas latitudes templadas su variabilidad característica y su muy oscilante tiempo atmosférico (Schaefer y Day, 1981).

\section{América del Norte: una región de constante oposición}

A diferencia de regiones tales como el subcontinente de Asia Sudoriental, donde las masas de aire que rigen el viento y el tiempo atmosférico son estacionales, América del Norte es una zona de constante oposición, donde cuatro tipos diferentes de masas de aire están siempre tratando de dominar el tiempo. Cada una de ellas no solamente lleva consigo un cambio de temperatura y humedad, sino que, en general, tiene también su propia pauta de vientos. El tiempo seco y estimulante característico del aire polar continental, llega generalmente con vientos del Noroeste que bar ren la neblina. En cambio, el aire marítimo trop i cal lleva consigo un estado húmedo y cálido, acompañado regularmente con vientos del Sur. El tiempo que se asocia al aire polar marítimo, muy probablemente vendrá con vientos del Norte, nubes bajas, niebla y lluvias que es el tiempo meteorológico ha bit ual en el Noroeste de México en verano. Las masas de aire trop ical continental que dominan en el Suroeste durante los meses cálidos, se distinguen por sus vientos relativamente ligeros (Schaefer y Day, 1981).

\section{México en el centro de la vorágine climática}

México, debido a su posición geográfica entre los $14^{\circ}$ y $\operatorname{los} 33^{\circ}$ de latitud septentrional, se encuentra comprendido, en su mayor parte, dentro de las zonas intertropical y subtropical del Hemisferio Norte, por lo que climáticamente hablando, su porción suriana viene a quedar en la zona de influencia de los vientos alisios, disfrutando de las características de las atmósferas barotrópicas existentes en las latitudes bajas con gran estabilidad meteorológica, al menos durante gran parte del año. El resto del país, al Norte del Trópico de Cáncer, se encuentra bajo la influencia desecante de los movimientos descendentes del aire, característicos de la zona de las altas presiones subtropicales, que son la causa de los grandes desiertos, no sólo de
México, sino de todo el Globo (Mosiño, 1974; García, 1978).

Durante la mitad caliente del año, con el desplazamiento hacia el Norte de la zona sub trop ical de alta presión, la región de los alisios aumenta en intensidad, latitud y altura, por lo que prácticamente todo el país queda bajo la influencia de estos vientos que soplan con una dirección de NE a SW en superficie, y de E a W en las alturas; éstos recogen humedad que introducen en las alturas a su paso por las aguas relativamente cálidas del Golfo de México, y posteriormente, la descargan en forma de lluvia sobre las pendientes montañosas que se inclinan hacia la planicie costera del Golfo de México, áreas directamente expuestas a los vientos húmedos del mar y al influjo de los ciclones tropicales en verano y a los "nortes" du rante el invierno (Ar royo et al., 1976). La parte más austral (Sur) de la República, queda bajo la influencia de la zona intertropical de convergencia (ZIC), que se desplaza también hacia el Norte en el verano (García, 1965 y 1978).

El patrón anterior de circulación gen eral de la atmósfera en la República Mexicana se ve modificado en la región de la planicie costera del Golfo de México por algunos factores del clima: la orografía, su cercanía con las aguas cálidas del Golfo de México y la amplia distribución lat i tu di nal sobre la que se extiende.

La orografía ocasiona un efecto de represamiento del viento, que implica el impedimento del paso de las corrientes hacia la porción in te rior del país por la presencia de la Sierra MadreOriental, sobre todo, en la temporada invernal, cuando se registran masas de aire polar o "nortes" provenientes de Estados Unidos y Sur de Canadá, que soplan violentamente durante varios días. Estas masas de aire se modifican al recoger humedad a su paso por las aguas cálidas del Golfo de México, para posterio rmente descargarla en forma de lluvia sobre las laderas de barlovento, es decir, de la parte expuesta al viento en la planicie costera. La cercanía del Golfo proporciona por una parte, humedad a los "nortes", y por otra, actúa como un amortiguador de los cambios térmicos, debido a la alta capacidad calórica del agua, lo que se refleja en una disminución de las oscilaciones de la 
La Oscilación del Atlántico Norte: un fenómeno que incide en la variabilidad ...

temperatura a lo largo del año (García, 1978; Mosiño y Morales, 1988).

\section{La Oscilación del Atlántico Norte (OAN): un fenómeno atmosférico con dos fases}

La Oscilación del Atlántico Norte es un subibaja a gran escala en la presión barométrica de las masas de aire atmosférico, situadas entre dos celdas: la de la alta presión subtropical del Atlántico (Azores), ubicada a los $38^{\circ}$ de latitud y la baja presión polar del Ártico (Islandia) a los $60^{\circ}$ de latitud. Esta oscilación tiene dos fases que se expresan mayo- ritariamente en invierno. La fase positiva sucede cuando las presiones barométricas del anticiclón de las Azores son más altas con respecto al valor promedio, mientras que las presiones en Islandia son aún más bajas de lo normal. Ésta marcada diferencia de presión intensifica los vientos del Oeste entre los 50 y $60^{\circ}$ de latitud Norte produciendo tormentas que cruzan el Atlántico en dirección Nordeste; tales tormentas transportan calor desde el Océano hasta la parte Nordeste de Europa, lo que ocasiona que el tiempo atmosférico en toda esa zona sea más suave y húmedo, al mismo tiempo que sobre la región mediterránea predomina la sequía (Figura 1a). 
N. Sánchez-Santillán, M. Signoret-Poillon y R. Garduño-López

Mientras eso sucede en Europa, el Noroeste de América se presenta más bien húmedo $y$, de manera paralela, en la Península del Labrador y en Groenlandia los tiempos atmosféricos son secos y fríos. Esto se debe a que los fuertes vientos del Noroeste viajan sobre el Mar del Labrador, causando un enfriamiento que provoca inviernos fríos y secos en Canadá y en Groenlandia. Este viento no pasa sobre el Mar de Groenlandia, por lo que esta región no se enfría demasiado, reduciéndose allí la severidad invernal (Hurrell et al., 2001; Wanner et al., 2001 y Rodríguez et al., 2004).
Du rante la fase negativa, las diferencias de presión entre las Azores e Islandia son menores. El "anticiclón" de las Azores está débil y la celda ciclónica de Islandia se encuentra poco activa; ambas se sitúan en latitudes más al Sur y, en consecuencia, los vientos del Oeste son más tenues y aportan menos humedad y calor sobre el Norte de Europa. Ambas celdas de presión, al localizarse más al Sur, ocasionan que la región del Mediterráneo se vea beneficiada de un tiempo menos seco. En el Noreste de América se registran inviernos más suaves y secos de lo normal (Figura 1b), (Hurrell et al., 2001 y Wanner et al., 2001). 
La Oscilación del Atlántico Norte: un fenómeno que incide en la variabilidad ...

Las modificaciones en el gradiente de presión entre una fase y otra producen grandes cambios en la velocidad y dirección de los vientos del Atlántico. El calor y el transporte de humedad entre este océano y los alrededores de los continentes americano y europeo varían marcadamente de acuerdo a la intensidad y número de tormentas invernales, sus trayectorias y el estado del tiempo meteorológico asociado a ellas. Asimismo, estos cambios en las presiones barométricas, acompañados de modificaciones en las temperaturas del Atlántico, alteran el nivel del mar, debido a que las masas de agua reaccionan a las variaciones en el régimen de los vientos, los cuales influyen sobre las corrientes, la altura y amplitud de las olas y la temperatura superficial oceánica (Hurrell et al., 2001 y Wanner et al., 2001).

La OAN constituye uno de los modificadores más importantes de la variabilidad atmosférica sobre el Norte del Océano Atlántico y juega un papel funda men tal en las variaciones climáticas en las porciones continentales del Este de América del Norte y en el continente Euroasiático, principalmente du rante los meses invernales.

\section{¿Qué controla a la OAN?}

Las investigaciones más recientes en torno a la OAN muestran qué intensidad de ésta puede ser trazada en mapas meteorológicos, no sólo en la superficie sino también en la troposfera y estratosfera. Asimismo, los cambios en la circulación del período invernal de la estratosfera baja en gran parte del Hemisferio Norte, muestran una tendencia al aumento de los vientos del Oeste que rodean el Polo Norte. Está bien establecido que la variabilidad de la temperatura y la presión barométrica en la troposfera puede manejar la variabilidad en la estratosfera, pero las nuevas observaciones y los modelos sugieren que parte de la estratosfera controla la troposfera.

Las tendencias de inviernos más severos observados en el clima de la superficie del Atlántico podrían estar ligadas a procesos que afectan la circulación de la troposfera en escalas de largo período. Las reducciones del ozono estratosférico y los incrementos en la concentración de gases de invernadero, contribuyen al enfriamiento radiativo de la estratosfera baja y media durante la noche polar, y son obviamente, candidatos para explicar el proceso. En una escala más corta, la estacional, un aparente retraso en la situación invernal de la troposfera responde a cambios en la circulación de la estratosfera y podría permitir el uso de una escala tem po ral intermedia para su predictibilidad (Hurrell et al., 2001 y Wanner et al., 2001).

El océano tiene también una influencia apreciable sobre la atmósfera. Las nuevas evidencias presentadas en los últimos congresos, muestran la tendencia de los océanos a preservar el estado térmico de un invierno al siguiente, al imprimir alguna continuidad de condiciones sobre la atmósfera, es decir, existe una memoria térmica (Adem, 1973; Greatbatch, 2000).

Los recientes análisis estadísticos, revelan patrones en las temperaturas superficiales del mar que preceden las dos fases específicas de la OAN con unos seis meses de anticipación (un proceso muy semejante al del ENOS) (Kim y Schneider, 2003).

Recientemente, los modelos de circulación general de la atmósfera donde se vinculan las temperaturas superficiales del océano y las cubiertas de hielo, confirman los patrones mencionados anteriormente, sobre todo, en los ciclos de la OAN multianuales y a más largo plazo, es decir, los interdecadales. Esto indica que la baja frecuencia de la variabilidad climática en el Atlántico Norte no es meramente un ruido en la estocasticidad de la atmósfera, sino más bien contiene una respuesta estructurada a los cambios en la temperatura superficial del océano o en la extensión del casquete polar.

Algunos investigadores vinculan las recientes tendencias de la OAN con el aumento progresivo de la temperatura superficial del océano tropical, en partic u lar, el observado en las aguas tropicales de los Océanos Índico y Pacífico, como consecuencia de las teleconexiones. Al deberse este calentamiento al incremento de los gases de invernadero, se estaría estableciendo la relación entre la OAN y las concentraciones del $\mathrm{CO}_{2}$ en la atmósfera. (Hurrell et al., 2001; Wanner et al., 2001; Jianping y Wang, 2002). 
N. Sánchez-Santillán, M. Signoret-Poillon y R. Garduño-López

\section{El surgimiento de la OAN como tema actual de la investigación científica}

Recientemente, se ha renovado el interés en la OAN básicamente por tres razones. La primera es que la OAN impacta fuertemente al Océano Atlántico induciendo modificaciones sustanciales en los patrones de los vientos superficiales; de ese modo, altera el intercambio de calor entre la superficie del océano y el agua contenida dentro de la atmósfera. Estas alteraciones afectan las características de la circulación termohalina del Atlántico y duplica el flujo hor izon tal de la superficie del océano, que podría revertirse temporalmente y hacerse más lenta, como lo predicen algunos modelos climáticos en respuesta a los cambios climáticos antropógenos (IPCC, 2001).

La segunda razón es que las alteraciones en la OAN tienen un amplio impacto sobre los ecosistemas marinos y terrestres, incluyendo la distribución a gran escala de las poblaciones de peces y crustáceos, la producción de zooplancton, el florecimiento de plantas, el crecimiento y reproducción de diversos animales terrestres (Bertacchi et al., 2000; Radi et al., 2001; Gibbins et al., 2002 y Hiscock et al., 2004).

La tercera causa es que un pequeño pero útil porcentaje de la variación de la OAN puede ser previsible. Esta predictibilidad puede surgir de la influencia de pequeños cambios en el océano, 0 motivada por la acción de factores externos, en par tic u lar, de los niveles ascendentes de los gases de invernadero, como sugieren las tendencias recientes del comportamiento de la OAN. Las posibilidades de pronóstico, aunque limitadas, contradicen la idea de que en la Oscilación del Atlántico Norte, los procesos atmosféricos que concurren y las resultantes de su interacción son de una naturaleza caótica tal, que hacen prácticamente imposible el conocimiento de su evolución presente y futura (Hurrell et al., 2001 y Wanner et al., 2001).

\section{Conclusiones}

Existen circulaciones atmosféricas de escala planetaria, tanto en la dirección me rid i o nal como zonal; asociadas a éstas últimas, se registran oscilaciones metereológicas de presión y temperatura de gran escala. Las dos más conocidas son El Niño-Oscilación del Sur y la Oscilación del Atlántico Norte. Consecuencia de esta estructura y funcionamiento del sistema climático, se dan las llamadas teleconexiones, es decir, los enlaces a grandes distancias entre anomalías climáticas aparentemente desconectadas entre sí. Naturalmente, nuestro país está expuesto a ambas oscilaciones, por cierto, una del Pacífico y otra del Atlántico.

Por to do lo an te rior, se vislumbra actualmente, por parte de la comunidad científica mundial y local, la necesidad de buscar posibles correlaciones entre ambas oscilaciones para explicar la variabilidad climática de México, así como su impacto en los sistemas naturales y artificiales. Una importante vertiente de estos estudios consiste en tratar de predecir esas oscilaciones con el consecuente impacto en nuestro clima, sobre todo, a la luz del calentamiento climático global de origen antropogénico, así como la forma en la que impactará en los sistemas antes mencionados.

\section{Referencias}

Adem J. (1973). Ocean Effects on Weather and Climate. Geofísica Internacional, No. 13, pp. 1-71.

Arroyo J., Serra S. y Estrada A. (1976). Atlas de trayectorias de ciclones tropicales. Centro de Ciencias de la Atmósfera, UNAM. México.

Bertacchi U., Tölle U. and Berndtsson R. (2000). Forecasting Discharge in Amazo nian Using Arti fi cial Neural Netwo rks. Inter national Journal of Climatology, Vol. 20, No. 12. pp. 1495-1507.

Cook E. (1992). Using Tree Rings to Study Past, EI Niño/Southern Oscillation Influences on Climate. En: El Niño: Histor ical and Paleoclimatic Aspects of the Southern Oscillation. Díaz, H. y Markgraf V. (eds.). Cambridge University Press, Inglaterra, pp. 203-214.

Díaz F. and Kiladis G. (1992). Atmo spheric Teleconnections Associated with the Extreme Phase of the Southern Oscillation. En: EI Niño: Histor ical and Paleoclimatic Aspects of the Southern Oscillation. Díaz H. y 
La Oscilación del Atlántico Norte: un fenómeno que incide en la variabilidad ...

Markgraf V. (eds.). Cambridge University Press, Inglaterra, pp. 29-68.

Durand-Dastes F. (1969). Climatología. Ariel. Colección ELCANO La geografía y sus problemas, Barce lona, pp. 334.

Flohn H. (1968). Clima y tiempo. Biblioteca para el hombre actual, Madrid, pp. 256.

García E. (1965). Distribución de la precipitación en la República M exicana. Boletín del Instituto de Geografía, UNAM, No. 1, pp. 175-191.

García E. (1978). A puntes de climatología. Offset Larios, México, p. 155.

Gibbins C., Moir H., Webb J. and Soulsby C. (2002). Assessing Discharge use by Spawning Atlantic Salmon: A Comparison of Discharge Electivity Indices and PHABSIM Simulation. River Research and Applications, Vol. 18, No. 14, pp. 383-395.

Glantz M., Katz W. and Nicholls N. (1991). Teleconnections Linking Worldwide Climate A nomalies. Cambridge Univer sity, pp. 535.

Greatbatch R. (2000). The North Atlantic Oscillation. StochasticEnvi ron mental Research and Risk Assessment, No. 14, pp. 213-242.

Hiscock K., Southward A., Tittley I. and Hawkins S. (2004). Effects of Changing Temperatureon Bentic Marine Life Britain and Ireland. A quatic Conser va tion: $M$ arine and Freshwater Ecosystems, Vol. 14, No. 4, pp. 333-362.

Hurrel J., Kushnir Y. and Visbeck M. (2001). The North Atlantic Oscil la tion. Science, Vol. 291, No. 5504, pp. 603-605.

IPCC. (2001). Climate Change. The Scientific Basis. Contribution of Working Group I to the Third Assessment Report of the Intergovernmental Panel on Climate Change. Cambridge Univer sity, pp. 881.

Jianping L. and Wang J. (2003). A New North Atlantic Oscillation Index and its Variability. Advances in Atmospheric Sciences, Vol. 20, No. 5, pp. 601-676.

Kim J. and Chneider R. (2003). Los-latitude Control of Interhemipheric Sea-Surface Temperature Contrast in the Tropical Atlantic over the Past 21 Kyears: the Posible Role of SE Trade Winds. Climate Dynamics, No. 21, pp. 337-347.

Mosiño P. (1974). Los climas de la República Mexicana. En: El escenario geográfico.
Introducción ecológica. Instituto Nacional de Antropología e Historia. Departamento de Prehistoria, México, pp. 56-172.

Mosiño P. y Morales T. (1988). Los ciclones tropicales, El Niño y las lluvias en Tacubaya, DF. Geofísica Internacional, Vol. 27, No. 1, pp. 61-82.

Radi T., Vernal A. y Peyron 0. (2001). Rela tion ships Between Dinoflagellate Cyst Assem blages in Surface Sediment and Hydrographic Conditions in the Bering and Chukchi Seas. Journal of Q uaternary Science, Vol. 16, No. 7, pp. 667-689.

Rodríguez-Fonseca B., Polo I., Serrano E. and De Castro M. (2004). A Subtrop ical Atlantic Predictor of Winter Anomalous Precipitation in the Iberian Peninsula, some European Regions and the North of Africa. Inter na tional Journal of Clima tology.

Sánchez-Santillán N. (1999). El cambio global de clima en el Golfo de México y Caribe Mexicano su repercusión en doce pesquerías de importancia comercial. Tesis Doctoral. Facultad de Ciencias, Universidad Nacional Autónoma de México, pp. 675.

Schaefer V. and Day J. (1981). A tmosphere. Clouds, Rain, Snow, Storms. Houghton Mifflin Company Boston, EUA, pp. 359.

Stephenson D., Wanner H., Brönnimmann S. and Luterbacher J. (2002). The History of Scientific Research on the North Atlantic Oscillation.In: The North Atlantic Oscillation: Climatic Significance and Environmental Impact (Hurrell J., Ottersen Y. y Visbeck M. Ed.) Geophysical Monograph 134, American Geophysical Union (AGU), Wash ington, pp. 37-55.

Wanner H., Brönnimann S., Casty C., Gyalistras D., Luterbacher J., Schmutz C., Stephenson D. and Xoplaki E. (2001). North Atlantic Oscillation-Concepts and Studies. Survey in Geophysics, No. 22, pp. 321-382. 
N. Sánchez-Santillán, M. Signoret-Poillon y R. Garduño-López

\section{Semblanza de los autores}

N orma Sánchez-Santillán. Realizó sus estudios en hidrobiología en la Universidad Autónoma Metropolitana, plantel Iztapalapa. Obtuvo el grado de maestra en ciencias (Biología), orientada en aspectos de climatología en la Facultad de Ciencias de la UNAM en 1994. Posteriormente, alcanzó el grado de doctora siguiendo la línea de aplicación climática en aspectos de ecología (1999). Cuenta con un diplomado en divulgación de la ciencia por parte de la Dirección General de Divulgación de la Ciencia, UNAM (2004). Es autora de 67 trabajos publicados en revistas y congresos nacionales e internacionales con arbitraje; así como 51 artículos de divulgación en revistas con arbitraje. Pertenece al Sistema Nacional de Investigadores desde el año 2002. Actualmente se desarrolla como investigadora en la UAM-Xochimilco como responsable del Laboratorio de Climatología, así como profesora titular dentro del plantel.

M artha Signoret-Poillon. Realizó sus estudios de licenciatura en biología en la Facultad de Ciencias de la UNAM. En 1972, obtuvo el doctorado en oceanografía en la Universidad de Aix-Marceille, Francia. La línea de investigación en la que se ha desempeñado es la biología marina, particularmente en plactología. Cuenta con la especialidad en formación para el ejercicio de la docencia, obtenida en el Centro de Investigaciones y Servicios Educativos de la UNAM, en 1982. Es autora de 26 artículos publicados en revistas internacionales y nacionales. Ha participado en 28 campañas oceanográficas y múltiples reuniones académicas nacionales e internacionales. Actualmente es profesora-investigadora titular en la Universidad Autónoma Metropolitana, Unidad Xochimilco, en el Departamento El Hombre y su Ambiente, asimismo, es responsable del Laboratorio Plancton y Bioenergética.

René G arduño-López. Obtuvo la licenciatura en física y el posgrado en geofísica por la Facultad de Ciencias de la UNAM. Ha sido profesor desde 1976, además de investigador titular y jefe del Departamento de Teoría del Clima del Centro de Ciencias de la Atmósfera, en la misma Universidad. Ha publicado artículos arbitrados en diversas revistas internacionales y ha presentado trabajos en decenas de congresos en México y en el extranjero; recopiló y editó la obra completa de J. Adem, publicada en varios volúmenes por El Colegio Nacional, es editor asociado de la revista especializada Atmósfera y autor de los libros "El veleidoso clima" (No. 127) y "Pormenores terrestres" (No. 183) de la Serie de la Ciencia para Todos, publicada por el Fondo de Cultura Económica y auspiciada por la SEP y el CONACYT. 